\title{
MARCO POLO: near earth object sample return mission
}

\author{
M. A. Barucci • M. Yoshikawa • P. Michel • \\ J. Kawagushi • H. Yano • J. R. Brucato • \\ I. A. Franchi • E. Dotto • M. Fulchignoni • \\ S. Ulamec • Marco Polo Science Team
}

Received: 16 November 2007 / Accepted: 10 March 2008/ Published online: 30 April 2008

(C) Springer Science + Business Media B.V. 2008

\begin{abstract}
MARCO POLO is a joint European-Japanese sample return mission to a Near-Earth Object. This Euro-Asian mission will go to a primitive Near-Earth Object (NEO), which we anticipate will contain primitive materials without any known meteorite analogue, scientifically characterize it at multiple scales, and bring samples back to Earth for detailed scientific investigation. Small bodies, as primitive leftover building blocks of the Solar System formation process, offer important clues to the chemical mixture from which the planets formed some 4.6 billion years ago. Current exobiological scenarios for the origin of Life invoke an exogenous delivery of organic matter to the early Earth: it has been proposed that primitive bodies could have brought these complex organic molecules capable of triggering the pre-biotic synthesis of biochemical compounds. Moreover, collisions of NEOs with the Earth
\end{abstract}

M. A. Barucci $(\bowtie)$

LESIA, Observatoire de Paris, 5, Place Jules Janssen, 92195 Meudon Cedex, France

e-mail: antonella.barucci@obspm.fr

M. Yoshikawa $\cdot$ J. Kawagushi $\cdot$ H. Yano

JAXA Space Exploration Center, Okinawa, Japan

P. Michel

Observatoire de la Cote d'Azur (OCA), Nice, France

J. R. Brucato

INAF-OAA, Florence, Italy

\section{A. Franchi}

Open University, Milton Keynes, UK

E. Dotto

INAF-OAR, Rome, Italy

M. Fulchignoni

University Paris Diderot, Florence, Italy

S. Ulamec

DLR, Köln/Berlin, Denmark 
pose a finite hazard to life. For all these reasons, the exploration of such objects is particularly interesting and urgent. The scientific objectives of MARCO POLO will therefore contribute to a better understanding of the origin and evolution of the Solar System, the Earth, and possibly Life itself. Moreover, MARCO POLO provides important information on the volatile-rich (e.g. water) nature of primitive NEOs, which may be particularly important for future space resource utilization as well as providing critical information for the security of Earth. MARCO POLO is a proposal offering several options, leading to great flexibility in the actual implementation. The baseline mission scenario is based on a launch with a Soyuz-type launcher and consists of a Mother Spacecraft (MSC) carrying a possible Lander named SIFNOS, small hoppers, sampling devices, a re-entry capsule and scientific payloads. The MSC leaves Earth orbit, cruises toward the target with ion engines, rendezvous with the target, conducts a global characterization of the target to select a sampling site, and delivers small hoppers (MINERVA type, JAXA) and SIFNOS. The latter, if added, will perform a soft landing, anchor to the target surface, and make various in situ measurements of surface/subsurface materials near the sampling site. Two surface samples will be collected by the MSC using "touch and go" manoeuvres. Two complementary sample collection devices will be used in this phase: one developed by ESA and another provided by JAXA, mounted on a retractable extension arm. After the completion of the sampling and ascent of the MSC, the arm will be retracted to transfer the sample containers into the MSC. The MSC will then make its journey back to Earth and release the re-entry capsule into the Earth's atmosphere.

Keywords Near earth object mission $\cdot$ Sampling $\cdot$ Sample return $\cdot$ Re-entry capsule

\section{Introduction}

Near Earth Objects (NEOs) are representative of the population of asteroids and dead comets that are thought to be the primitive leftover building blocks (embryos) of the Solar System formation process. Thus, they offer clues to the chemical mixture from which the planets formed some 4.6 billion years ago. They carry records both of the Solar System's birth/early phases and of the geological evolution of small bodies in the interplanetary regions [13].

In contrast to the planets, which underwent evolutionary processes during their history, most asteroids and (dormant) comets, due to their small sizes, are believed to have retained a record of the original composition of the proto-planetary disk in which they were formed [44]. Thus, they can be considered as the DNA of the Solar System which can give us some hints to the origin of planets and life. While comets come from larger distances from the Sun $[14,46]$, the majority of asteroids reside in a broad band between the orbits of Mars and Jupiter, called the "main-belt". A distinct population of objects, which originates mainly from the main asteroid belt, consists of small bodies whose orbits cross those of the terrestrial planets and is called the Near Earth Object (NEO) population.

NEOs are asteroids and comet nuclei in an evolving population with a lifetime limited to a few million years after which most of them end in a Sun-grazing state, or are ejected from the Solar System, while about 10-15\% of them collide with a terrestrial 
planet. However, despite the short dynamical lifetime of their orbits, their number is maintained in a steady-state. Indeed, most NEOs come from different zones of the main belt, via specific mean motion and secular resonances with Jupiter, with a possibly significant contribution of extinct cometary nuclei. The Mars-crosser population originating from high diffusive regions of the main belt, serves also as an intermediary source of a great fraction of large $(>5 \mathrm{~km})$ NEOs [32]. NEOs, in turn, are supposed to be one of the principal sources of meteorites found on Earth. Up to June 2007, more than 4500 NEOs of all sizes have been discovered, and the entire population is estimated to contain more than 1000 objects with diameters larger than $1 \mathrm{~km}$ and hundreds of thousands greater than $100 \mathrm{~m}[33,45]$. In contrast, meteorites in our collections appear to originate from a much smaller number of bodies, of the order of 100, and the link between asteroid types and meteorite samples on Earth remains tenuous.

The small body population is considered to be a continuum of leftover planetesimals whose principal differences arise from the variety of formation regions and evolutionary histories [26]. NEOs are a precious source of information as they represent a mixture of the different populations of small bodies, i.e. main-belt asteroids and cometary nuclei, and allow a link with meteorites. Examples include the identification of the asteroid (4015) 1979 VA as actually being the extinct comet $107 / \mathrm{P}$ Wilson-Harrington, and the discovery of 3200 Phaeton as the parent body of the Geminids meteor stream [47].

Most of what we know about small bodies has been acquired through observations from the ground and from the study of meteorites delivered to the Earth's surface [7, 21]. However, Earth-based observations are not powerful enough to fully characterize the wide diversity of the population.

In-situ measurements provide more suitable critical information. Several space missions have included a fly-by of an asteroid or of a comet (see Fig. 1). Only two missions have been specifically devoted to a rendezvous with a NEO, namely the NASA NEAR Shoemaker and the JAXA Hayabusa missions. The NASA Stardust mission is successful fly-by collected thousands of micron and submicron-sized cometary dust particles for laboratory analysis.

Fig. 1 Size vs. albedo plot of the small bodies visited by space missions. With the exception of Mathilde, all the visited asteroids belong to the higher albedo $\mathrm{S}$ class

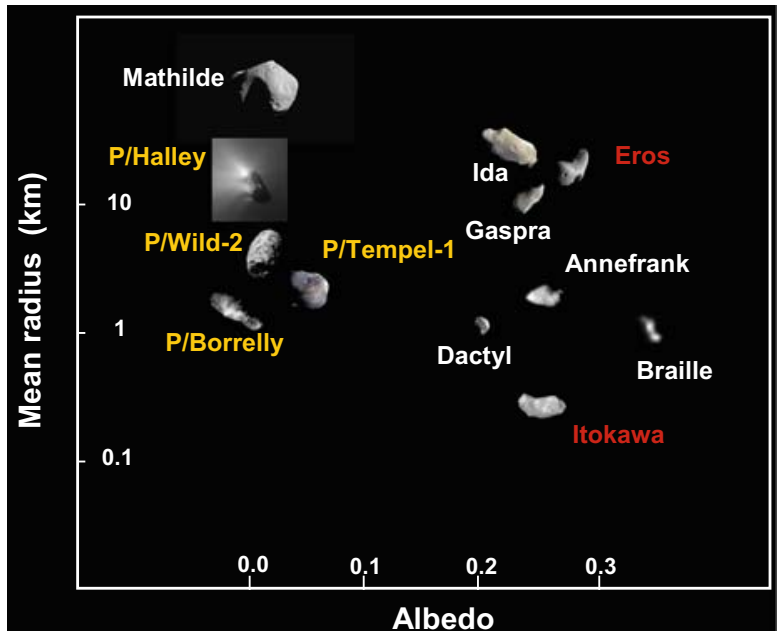


The population of the main belt asteroids (MBAs) presents a high degree of diversity as revealed by ground-based observations. More than 10 major spectral classes have been identified [2, 8, 9]. These taxonomic classes group objects with similar spectral properties, supposing similar surface composition, and probably similar evolution. The most intriguing are the dark main belt asteroids (MBAs), such as the C-, P- and D-types, as they are considered among the most primitive asteroids. Low albedo asteroids are widely believed to have preserved material which witnessed the condensation and the early phases of the formation of the Solar System.

Unfortunately, only bulk spectral information has been gathered so far about the surface composition of NEOs, and refers to a sampling of only $\approx 10 \%$ of the 4500 known NEOs. The most evident property is the variety of spectral features, physical characteristics, and compositions: the NEO population includes all the taxonomic classes present in the main belt. Binzel et al. [4] carried out a wide analysis of the source regions of NEOs and concluded that the $v_{6}$ secular resonance is the most important source for observed NEOs belonging to most taxonomic classes. They also showed that C-type NEOs have an origin from the mid to outer belt, P-type objects from the outer belt, D-type NEOs from the Jupiter-family comets.

Although good spectral matches among some NEOs and meteorite types have been found (e.g. [27]), the link between NEOs and meteorites is not completely understood. More than about 35000 different meteorites now exist in collections across the world. However, we have strong suspicion that our terrestrial record is biased. For instance, although carbonaceous meteorites belonging to the so-called CM class constitute by far $(\sim 35 \%)$ the majority of carbonaceous chondrites, it is possible that they come from one single asteroid only [34]. Moreover, only the strongest material can survive atmospheric entry [11], and it is not known whether this material is representative of the dominant material in space. For instance, the measured compressive strength of the Murchison meteorite is $50 \mathrm{MPa}$, which is much higher than the compressive strength of porous materials on Earth. This could explain the apparent over representation of "ordinary chondrites" in the meteorite collections compared to dominant interplanetary matter. Based on the lunar experience, the "space weathering" effects of impact processing and solar wind irradiation on the surface of atmosphereless small bodies are known to have a significant influence on the reflectance spectra of the surface regolith (e.g. [10]). Therefore, attempts to match the reflectance spectra of meteorites with those from asteroids return poor or ambiguous results.

\section{Scientific objectives}

A mission to a primitive NEO (e.g. dark D, P or C-type) can provide crucial elements to answer the following key questions.

\subsection{What were the processes occurring in the primitive Solar System} and accompanying planet formation?

The Solar System formed from a disk of gas and dust orbiting around the Sun. From meteoritic studies it would appear that the formation of the Solar System, from 
collapsing nebula to planetary embryos, was a rapid process - lasting at most a few million years [37]. The chronology of these events is still poorly understood. Subsequently, once the first planetesimals were formed, a runaway growth occurred, in which the largest planetesimals started to accrete mass from the smaller objects, growing bigger and increasing the relative mass difference with the remaining objects. Thus, the planetesimals could represent the building blocks of the planets [15], and in this respect their analysis is expected to bring us crucial information on the nature of the protoplanetary disk [22, 25, 29].

We can already note that returned primitive material from a small body offers the possibility to identify nebula effects and resolve them from asteroidal processes. Primitive objects include material originating from or modified by stellar outflows, the interstellar medium, the solar protoplanetary disk, and the parent-body processing. Because large-scale mixing was a major phenomenon in the early Solar System [6], they include materials formed in different regions of the solar nebula and at different times under very different physico-chemical conditions [5, 12]. The isotopic composition of various elements, the nature of the organics and the mineralogy of the rocky elements in primitive Solar System bodies are requisite data to obtain information on the great variety of processes that took place during Solar System history.

Unaltered material also permits determination of the abundance of a number of short lived radio-nuclides present at the time of formation of a variety of early solar nebula components - essentially free from the concerns of partial re-setting or secondary process effects - offering a clear insight into the timing of the formation of these components and determining whether they have a local (e.g. irradiation and ejection by $\mathrm{X}$-wind or other processes [12]) or remote (e.g. stellar nucleosynthesis) origin.

\subsection{Do NEOs of primitive classes contain presolar material yet unknown in meteoritic samples?}

One of the major achievements in meteoritics over the past 20 years has been in the isolation and detailed analyses of a wide range of different pre-solar grains found in the primitive meteorites (e.g. [35]). The latest, and potentially the most important group of grains identified in meteoritic material are the interstellar silicates, but these are only found in specific areas of matrix composed of very fine-grained anhydrous phases (e.g. [36]). To date very few have been found where aqueous alteration has been prevalent, highlighting the susceptibility of these grains to processes occurring on the parent asteroids - particularly the effects of water. Similarly, the abundance of other, rarer presolar grains such as nanodiamond, $\mathrm{SiC}$ and graphite all show marked decreases in abundance with increasing metamorphism and/or aqueous alteration [18-20]. Primitive material originating from near the surface of an asteroid should contain abundant presolar grains, particularly silicates. This offers the opportunity to investigate the abundance of such grains accreted to the parent body and to search for new, less robust grains which have not survived the meteorite formation processes.

The latest techniques now provide the opportunity to investigate the sub-micron margins or mantles of these micron sized grains (e.g. [3]), which record a wealth of information about the environments and processes the grains have experienced since their formation - offering insight into the ISM and early nebula. However, by their 
very nature these rims or mantles are likely to be particularly susceptible to modification or destruction during meteorite formation on the parent body, processes experienced by meteorites. Once again, primitive material collected from the surface of a NEO offers the best opportunity for obtaining pristine grains.

2.3 How did asteroid and meteorite classes form and acquire their present properties? How do asteroids and meteoritic classes relate to each other?

Because meteorites fall randomly on Earth, it is very difficult to unequivocally determine whether any two meteorites originated from the same object. Such pigeonholing, as established with detailed mineral chemistry and oxygen isotopic measurements, is actually very important when attempting to determine which meteorites may have originated from common asteroids - a knowledge which is critical when attempting to unravel the complex origin and formation histories of individual asteroid types. Samples from a known, and in this case single, locality eliminate this uncertainty and discussion.

2.4 What is the link between the vast array of spectral information on asteroids and the detailed knowledge available from meteorites?

Considerable effort has been made to match reflectance spectra obtained from asteroids with known samples of meteorites. Good matches have been achieved for highly evolved (melted) bodies (e.g. 4 Vesta and the basaltic achondrites [23]), but become increasingly more tenuous with decreasing albedo (increasing organic content) and other characteristics of more primitive mineralogy. A significant complication is that space weathering (from solar wind irradiation and impact processing) has a major effect on the surface properties of airless bodies. The effects of space weathering are very difficult to simulate in the laboratory, but have been studied in great detail using returned lunar samples (e.g. [40]). However, the composition and space environment of the lunar surface is quite different to that of asteroids - therefore it is unclear whether the lack of key space weathering components in meteorite regoliths such as glassy agglutinates and nanophase iron is a result of preservation or weathering processes (e.g. [38]).

Interpretation of all remote observation data is greatly enhanced by "ground truth" samples. Laboratory reflectance spectra of individual components from a returned sample of a primitive NEO can be compared with telescope spectra. The level of space weathering each component has experienced can also be determined mineralogically and geochemically (e.g. noble gas studies), together with the detailed comparison of mineralogy and chemistry with known meteorite types. Only with such work will it be possible to apply the detailed knowledge obtained from meteorites to the vast amount of information available from asteroid observations.

2.5 What are the main characteristics of the internal structure of a NEO - both physically and chemically?

Remote sensing and physical parameter measurements from a hovering or orbiting position combined with a sample or samples of mixed regolith from the surface of an 
asteroid can offer a clear insight into this problem. The gravitational potential and inhomogeneities within the volume of the object can be obtained by remote sensing measurements, and with higher accuracy using radar tomography. Furthermore, impact processing on the asteroid will result in significant amounts of interior material distributed all over the surface - and therefore even a decent grab sample of well mixed regolith would be sufficient to derive some indications on the mineralogy, composition, chronology and history of a given asteroid.

2.6 What is the nature and origin of organic compounds on a NEO? How do NEO organics shed light on the origin of molecules necessary for life? What is the role of NEO impacts in the origin of life on Earth?

Current exobiological scenarios for the origin of life include invoking an exogenous delivery of organic matter to early Earth [11]. It has been proposed that carbonaceous chondrite matter (in the form of planetesimals or cosmic dust) could have brought these complex organic molecules capable of triggering the prebiotic synthesis of biochemical compounds on early Earth (e.g. [31] and references therein). However, those meteorites with abundant organics also display the highest levels of aqueous alteration, and it is clear that this has had a major impact on the nature of the organics present, modifying or destroying those formed in earlier events [30].

The low albedo of primitive asteroids, e.g. C, D, P types, is inferred to be the result of abundant organic matter present on the surface of the asteroids - indicating that these bodies have experienced little or no thermal processing. However, some of these bodies do appear to have experienced some kind of aqueous alteration process, with $\approx 60 \%$ of the C-type asteroids, at heliocentric distances between 2.5 and $3.5 \mathrm{AU}$, showing spectral features indicating such activity [1]. The D type asteroids, on the other hand, may be predominantly composed of anhydrous minerals and organic matter - indicating that the surfaces of these bodies have not experienced any significant aqueous activity.

With regard to meteorite organic matter, the major asteroidal carbonaceous component will likely be an insoluble organic matter, whose origin in carbonaceous chondrites is still largely debated (e.g. [43]). The nature of its formation processes, together with insight into the precursor material, can be obtained through techniques such as structural analysis (e.g. ${ }^{13} \mathrm{C} \mathrm{NMR}$ ) and identification of the abundance patterns of the different homologous series within the fractions liberated from pyrolysis and other degradation experiments (e.g. hydropyrolysis). This is particularly powerful used in combination with the stable isotopic measurements of individual compounds within each series, and compared to any co-existing free compounds present in the samples [16].

Current investigation of the most primitive organic materials available from samples such as the Stardust cometary samples and the IDPs are limited to a few techniques - offering exceptional spatial resolution or sensitivity, but lacking in the detailed abundance and isotopic information available from the meteorite samples.

One of the most important observations to date has been the identification of chiral excesses in $\alpha$-dialkylamino acids in the soluble fraction of the meteoritic organic matter (e.g. [41]). It was demonstrated that some of the most abundant 
amino acids display an excess of the left-handed version (L-enantiomer) over the right-handed version (D-enantiomer) of up to $15 \%$, called enantiomeric excess (ee). Due to their molecular architecture, these amino acids are stable against a process called racemization, which leads to an equilibration of left- and right-handed enantiomers of these molecules. Other amino acids, which could have been affected by terrestrial contamination, have been shown to have much lower ee's of $1-2 \%$. It has been suggested that the observed preference for left-handedness may be related to the left-handedness that is the signature of Life on Earth, strengthening the possibility that the meteoritic organics had a role in the origin of Life on Earth [42]. Understanding the origin of the amino acids and their distribution in the early Solar System will contribute to assessing the likelihood of this scenario, and indeed its applicability on other planets or exoplanets.

A sample from a primitive NEO containing a number of components with varying degrees of low levels of aqueous alteration (monitored via the silicate mineralogy) would give some definitive answers on the formation processes of carbonaceous matter in interplanetary material, including key biological compounds like the amino acids. By returning samples free from contamination the ambiguity created by terrestrial contamination is eliminated.

\subsection{Why are the existing meteorite specimens not suitable?}

All meteorites must survive atmospheric entry. Therefore, unsurprisingly, all meteorites are very tough, coherent rocks. Such strength is the result of metamorphism, shock and/or aqueous alteration on the parent asteroids - with effects that extend well beyond the mechanical properties of the meteorites as they mobilise elements and isotopic ratios within and between minerals, re-set radioisotope chronometers, destroy and modify primitive materials, and synthesise and mobilise organic compounds. IDPs, micron-sized fluffy dust grains, display mineralogical, chemical and isotopic signatures, not found (destroyed?) in meteorites, that strongly indicate formation and/or residence in the ISM or solar accretion disk (e.g. [24]). Such primitive material must have been stored somewhere for the past $4.5 \times 10^{9}$ years. It may have been stored in comets. Although the Stardust samples from comet Wild 2 have failed to reveal many of the primitive features seen in IDPs, the Stardust samples were heavily altered during impact in aerogel (e.g. [48]). On a more macroscopic scale, the Tagish Lake meteorite has a significantly lower mechanical strength than other carbonaceous chondrites. This friable meteorite appears particularly primitive, with high carbon content and unusual organic inventory, with a possible association to D type asteroids [17]. These rare and unique samples demonstrate that mechanically weak material does exist in significant quantities within the inner Solar System and that the existing meteorite collection is strongly biased towards more heavily processed material.

\subsection{Why do we need to return a sample to Earth?}

Many of the science questions we are attempting to resolve stem from detailed knowledge obtained from high precision and sensitivity measurements of meteorite properties. To advance the science with the new samples anticipated from a primitive 
asteroid will require comparable levels of analytical capability - attempting to do so in different ways would only lead to ambiguous results. The ability of in situ or remote sensing instruments to emulate lab-based instruments in providing high sensitivity, high precision or high spatial resolution measurements is compromised by constraints due to limitations of size, mass, power, data rate, and reliability imposed by the practical aspects of space missions. Many of the answers to the scientific questions will only be obtained through sophisticated analysis chains and integrated studies. Examples of this need are highlighted by the studies of the organic materials. From a coarse regolith specific lithologies will need to be isolated. The actual organic analyses will require precise (e.g. percent level) abundance measurement of low presence (e.g. nmol/g) of free compounds and their isotopic composition ( $\mathrm{C}, \mathrm{N} \leq 1 \%, \mathrm{D} / \mathrm{H} \leq 20 \%$ ) to determine their origin and formation.

Understanding the origin and formation of the organic compounds requires studying a wide range of moieties encompassing key stages of the most likely reaction pathways. Probably the most abundant organic material will be the very complex insoluble macromolecules, requiring a wide range of analytical tools to be identified, linking back to the mineralogical features at the micron level.

Critical to virtually all the key mineralogical, chemical and isotopic analyses to be performed is the selection of the correct sub-sample and the exact preparation requirements. Perhaps the most demanding measurements are those associated with the chronology of the samples. Whether attempting to date original accretion disk events or secondary minerals formed in the parent body these are essential for understanding the overall petrographic context, permitting correct interpretation of the data. Meteoritic data indicate that the main aspects of Solar System formation occurred in less than a few million years and therefore to achieve the required $<1$ Myr temporal resolution, analytical instruments with exceptionally high precision and sensitivity are required. The challenge of working with small amounts of material, constructing complex analysis sequences and interpreting the results is a routine feature of modern meteorite and IDP investigations, and also draws upon specific sample return experience with the Stardust and Genesis samples. The analysis of samples, returned to Earth and stored on the ground, will benefit from future development in analytical techniques. Thus, a sample return mission can provide significant scientific results far beyond the actual mission duration.

In conclusion, Near Earth Objects (NEOs) have the advantage of being much more accessible for scientific research and space missions than small bodies in other populations (comets and main-belt asteroids). A space mission to a NEO thus provides major opportunities for advancement in our understanding of some of the fundamental issues on the origin and early evolution of the Solar System, and for investigating the primordial cosmochemistry of the solar protoplanetary disk and the formation and properties of the building blocks of terrestrial planets.

Moreover, considering the threat represented by those NEOs classified as potentially hazardous objects, knowledge of the physical properties of NEOs is critical in determining their behaviour during atmospheric entry and in deciding whether the object will break-up before reaching the ground. The identification of the true composition and internal structure of a NEO is also the first essential step towards developing means to deflect or destroy an object whose trajectory leads to a collision with Earth. 


\section{Mission target selection}

NEOs are among the most accessible bodies of the Solar System. Several hundreds of NEOs are accessible with delta-V $<6 \mathrm{~km} / \mathrm{s}$. Starting from the classical definition of "accessibility" of a celestial body in terms of the velocity change (delta-V) needed to realize a rendezvous mission (e.g. orbiting around an object), it is in fact possible to show that NEOs can be more accessible than the Moon (Fig. 2). The current number of easily accessible objects will certainly increase. Observational campaigns, dedicated to those objects will characterize among them the scientifically interesting targets for in-situ and/or sample return missions.

Each target has flexible launch windows and short mission durations.

A number of possible targets of high scientific interest have been selected covering a wide range of launch windows in the time span 2017-2019, with mission duration from about 4 to 8.5 years. Among them:

- the dormant comet 4015 Wilson-Harrington, which can provide insights into the origin and evolution of comets transported in the near-Earth space as well as the unknown link between asteroids and comets;

- asteroids belonging to the D-type class, such as $2002 \mathrm{AT}_{4}$, or $2001 \mathrm{SG}_{286}$; a particularly primitive asteroid type offering excellent samples of the inner solar nebula

- the C-type double asteroid $1996 \mathrm{FG}_{3}$, another type of primitive asteroid that also offers the opportunity to provide insight into binary formation processes.

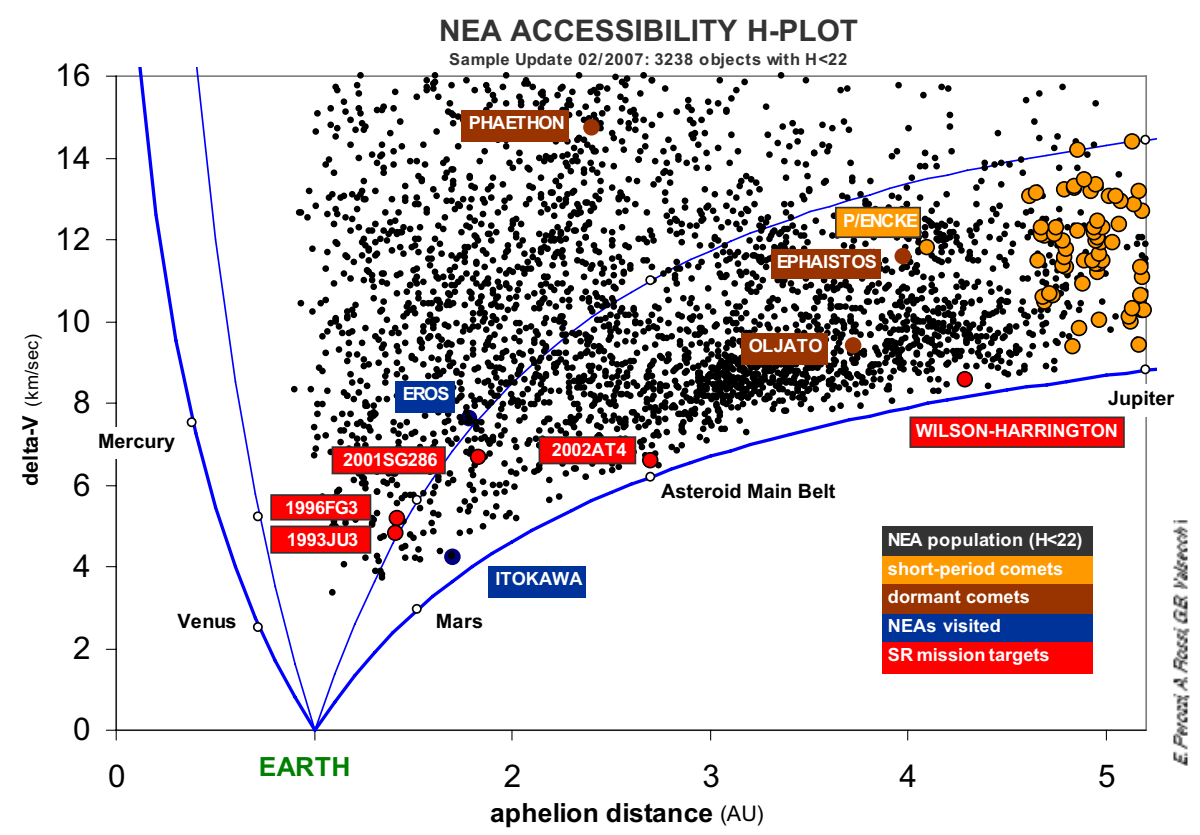

Fig. 2 The accessibility of NEOs versus required delta-V (adapted from [39]) 
All of these objects are likely comprised, at least in part, by materials that rarely (if ever) survive passage through the Earth's atmosphere as meteorites and should be some of the most primitive materials available to study early Solar System formation processes. Most importantly, MARCO POLO will provide samples from a known target and known geological context - crucial scientific factors that are never known for meteorites. The samples will also be collected under controlled, clean conditions that can never be rivalled by randomly falling meteorites - providing a unique opportunity to investigate the nature of the complex organic material present without interference from terrestrial contamination and therefore probe the importance of this material to the origin of Life on Earth and potentially elsewhere in the Solar System and beyond.

\section{Mission profile}

All selected targets require a Soyuz-Fregat launcher (ESA) to be inserted in to an initial heliocentric orbit for EDVEGA (Electric Delta-V Gravity Assistant) to commence the interplanetary cruise phase. MARCO POLO is a flexible mission with various technical options and targets. The choice of the target depends on a trade-off between science output and available resources.

The baseline mission scenario includes a launch with a Soyuz-type launcher of a Mother Spacecraft (provided by JAXA, hereafter MSC), sampling devices (ESA and JAXA), a re-entry capsule (ESA and JAXA) and scientific payloads (shared between Japan and Europe). The MSC leaves Earth orbit, cruises toward the target with ion engines, rendezvous with the target, conducts a global characterization of the target to select a sampling site, and delivers small hoppers (MINERVA type, JAXA). The possibility to carry a Lander (provided by ESA) has been also studied. The Lander itself profits considerably from the Rosetta Lander (Philae) heritage. The latter will perform a soft landing, anchor to the asteroid surface, and perform various in situ measurements of the surface/ subsurface material near the sampling site. The sharing between ESA and JAXA shall be analyzed in more detail during the assessment study at ESA with JAXA.

Two surface samples will be collected by the MSC using a "touch and go" manoeuvre. Sample containers will then be inserted into the re-entry capsule. The MSC will return towards the Earth and will release the capsule for the high-speed reentry into Earth's atmosphere. The capsule will be retrieved on the ground at a low to mid latitude, non-habited area, possibly in the northern hemisphere. After appropriate space quarantine and sterilization processes, samples will be taken out of the capsule in a dedicated curation facility to conduct initial sample characterization, prior to their distribution to designated scientists for detailed analyses. All the scientific data acquired and analytical results of the returned samples will be jointly archived by ESA and JAXA for public release after the proprietary period.

The most challenging mission scenario to 4015 Wilson-Harrington, is summarized in Table 1. After the cruise phase with ion engine acceleration, the spacecraft will rendezvous with the target and follow it in its journey around the Sun. From this "hovering" position, the MSC will start to characterize the global properties of the target with onboard remote sensing scientific instruments in order to determine in 
Table 1 A baseline MARCO POLO mission scenario to 4015 Wilson-Harrington

\begin{tabular}{|c|c|c|}
\hline Dates & Events & Notes \\
\hline $2018 / 04 / 25$ & $\begin{array}{l}\text { Soyuz-Fregat launch MSC total } \\
\text { mass } 1320 \mathrm{~kg}\end{array}$ & $\begin{array}{l}\text { Inserted to interplanetary orbit for EDVEGA } \\
\text { acceleration (first back-Up Window in '18/10) }\end{array}$ \\
\hline $2019 / 10 / 10$ & Earth gravity assist (Swing-by) & IES acceleration \\
\hline $2022 / 09 / 24$ & Rendezvous with Wilson-Harrington & At $1.04 \mathrm{AU}$ after perihelion passage \\
\hline \multirow[t]{6}{*}{ 2022/09-12 } & Global characterization & Staying at the target for $\sim 100$ days \\
\hline & Sampling/landing site selection & \\
\hline & Touchdown rehearsals & \\
\hline & Lander deployment and measurements & Few days \\
\hline & Touch-and-Go sampling & \\
\hline & IES restart tests & \\
\hline $2023 / 01 / 01$ & Departure from Wilson-Harrington & IES operation restart at $1.7 \mathrm{AU}$ \\
\hline $2026 / 10 / 01$ & Earth return and capsule retrieval & Re-entry velocity at $\sim 14 \mathrm{~km} / \mathrm{s}$ \\
\hline
\end{tabular}

particular a three dimensional shape model and its gravitational field. Moreover, surface morphology at global scale will be investigated to select the preferred landing and sampling sites.

During the descent sequence, small hoppers (MINERVA-type) will be released while the larger Lander SIFNOS, if added, will be deployed to the selected site. SIFNOS will perform a soft landing, anchor to the asteroid surface and make various in situ measurements of surface/subsurface material near the sampling site with on board instruments within several Earth days. Two surface samples will be collected by the MSC using a "touch and go" manoeuvre. Two complementary sample collection devices will be used, developed by ESA (sticky pad) and JAXA (Hayabusa heritage), respectively, mounted on a retractable extension arm. After the completion of the sampling and ascent of the MSC, the arm will be retracted to transfer the sample containers into the MSC.

For the proposed baseline (and most challenging) target, Wilson-Harrington, we have designed a mission scenario with launch in April 2018 (possible back up in late 2018 and 2019).

Table 2 shows three alternative mission scenarios for the other three possible targets.

Table 2 Possible mission scenarios for the other three target candidates, all based on a launch with Soyuz Fregat

\begin{tabular}{llll}
\hline & $2002 \mathrm{AT}_{4} \mathrm{D}$ type & $2001 \mathrm{SG}_{286} \mathrm{D}$ type & $1996 \mathrm{FG}_{3} \mathrm{C}$ - binary \\
\hline Launch Date & $03 / 232017$ & $06 / 132017$ & $07 / 202017$ \\
$\mathrm{~V}_{\text {inf } \mathrm{km} / \mathrm{s}}$ & 3.5 & 4.1 & 3.46 \\
Escape. Mass & 1320 & 1320 & 1320 \\
RdV Date & $06 / 062020$ & $06 / 152019$ & $04 / 192021$ \\
Departure Date & $09 / 062020$ & $09 / 152019$ & $07 / 192021$ \\
Departure mass & 1057 & 1048 & 1090 \\
Re-entry Date & $03 / 292024$ & $06 / 232022$ & $05 / 162022$ \\
Return $\mathrm{v}_{\text {inf }} \mathrm{km} / \mathrm{s}$ & 6.0 & 6 & 8.35 \\
Mission Duration & $\sim 7$ years & $\sim 5$ years & 4.8 years \\
\hline
\end{tabular}


The mission scenario depends on the orbital properties of the target of the mission. As those properties are different for the three targets, our mission can offer different launch opportunities and mission durations, depending on which target is eventually chosen. This is a big advantage of such a space mission, which offers considerable flexibility in case some unexpected changes in the programme occur (as happened for the Rosetta mission).

Launch dates have been determined to be compatible with the programme frame, given by Cosmic Vision. For each target many other launch windows were possible, and other scenarios could be defined later to adapt to a potential change of programme. So, these scenarios should not be considered the unique options. Depending on the target, the mission can last from 4 years to 8 years. The overall spacecraft mass is also constrained by the target choice. The assumption on the engine performances and characteristics used to define these scenarios are compatible with the ones that will be available for the mission.

The ground segment can be classical including a flight dynamics operation centre (JAXA \& ESA), using a very representative simulator with an important NEO environment modelling component. The connection to ESA deep space network associated with JAXA antenna of Usuda Deep Space Center will be used during specific phases of the mission (RdV, landing, sampling and Earth re-entry).

\section{Payload concept}

The sample return mission design includes the MSC and possibly the Lander SIFNOS. To reach the scientific goals of the mission we propose a multi-scale approach according to the spatial range of analyses: macroscopic global scale $(\mathrm{km}$, $\mathrm{m})$ will be reached by the MSC and the local scale $(\mathrm{cm}, \mathrm{mm}$ ) will be reached by the Lander, while the microscopic scale $(\mu \mathrm{m}, \mathrm{nm})$ will be reached by laboratory analyses of returned samples. The global characterization of the target (e.g.: size, shape, mass, internal structure, etc.) is crucial to link the collected samples with the origin and evolution of the parent object. Furthermore, landing site and sample return decisions require characterization of the object obtained both by remote measurements performed with payload instruments on board the MSC and by the Lander with selected instruments that will perform local in situ measurements, possibly at several surface locations. The MSC scientific payload includes a high resolution imaging system, spectrometers covering visible, near-infrared and mid-infrared wavelengths, a laser altimeter, a dust monitor and a radio science experiment. These instruments will be operated during the approach, hovering and descent phases and are essential for landing site selection, sample context characterization and for spacecraft safety. Other instruments, in secondary priority, can be added during the assessment phase study, as the Radar, X-Ray Spectrometer, Solar Monitor, Gamma-ray spectrometer, Neutron counter, and Neutral Particle Detector.

Instruments on the Lander (e.g., close-up/panoramic camera, electron microscope, X-ray diffractometer, volatile detector, microbalance, mass spectrometer, and thermal sensors, with first priority) would allow characterizing the location, context, and surface environment of the sampling site. Other instruments, such as mid-infrared spectrometer, Raman microscope, gamma-ray spectrometer, alpha 
particle X-ray spectrometer, surface package, electric field sensor can also be added.

A firm strawman payload should be defined after detailed study, depending on the chosen mission option and target.

Any sample collected at or near the surface of a NEO will be a complex mixture of different components with different compositions and morphologies. The sample could have suffered intense space weathering. Such weathering can modify the sample's chemical and mineralogical properties or form micro-structures that require analyses in situ. It is anticipated that the return-sample collection techniques will provide limited depth information - and therefore in order to understand the nature of the collected materials detailed characterisation of the sample collection site is required - this can only readily be achieved by use of a Lander with a comprehensive instrument package deployed in the vicinity of the actual collection site. The data provided by the Lander will provide information on a scale intermediate $(\mathrm{mm}$ to $\mathrm{cm}$ ) to that obtained by the MSC and the returned samples. Indeed, an accurate knowledge of the local context where the samples come from is fundamental to describe their distinctive nature.

A detailed microscopic study with very high levels of analytical precision of the material collected at the surface or sub-surface is also needed, and can only be achieved in laboratory.

In summary, the key measurements to be performed on or near the surface of the target body are:

Global-scale:

- Overall characteristics: orbit, rotation, size, shape, mass, gravity and density.

- Surface topography and morphology (boulders, craters, fractures,).

- Main characteristics of the internal structure.

- Mineralogical and chemical compositions.

- Dust conditions around the object.

Local-scale:

- Mineralogical composition and crystal structure of surface minerals.

- Out-gassing volatiles (e.g. $\mathrm{H}_{2} \mathrm{O}, \mathrm{CO}_{2}, \ldots$ )

- Complex organic molecules.

- Surface thermal properties.

Moreover a sample collector is requested for the sampling and a robotic arm onboard the Lander could be suitable for multiple in-situ analyses.

The MSC and Lander strawman payload are reported in Figs. 3 and 4.

As requested by ESA for an $\mathrm{M}$ class mission, the proposed instruments are based on existing/under development technologies (TRL $\geq 4)$.

A Robotic Arm (RA) can be used to position instruments into direct contact with the soil. A very light RA taking the instruments to the soil allows the possibility to analyze the sample in its original position and to avoid any sample contamination as well as to correlate environmental conditions. For this type of arm, preliminary budget allocation are: mass $2 \mathrm{~kg}$; volume $450 \times 200 \times 150 \mathrm{~mm}^{3}$ and power $<10 \mathrm{~W}$. Indication of volume and mass allocated for the instruments on the wrist are $\approx 2.2 \mathrm{~kg}$ 
Fig. 3 Main Spacecraft instruments which will allow to meet the scientific objectives. In black the primary payload, in blue the secondary choice, the Lander can be considered as facility or consortium payload

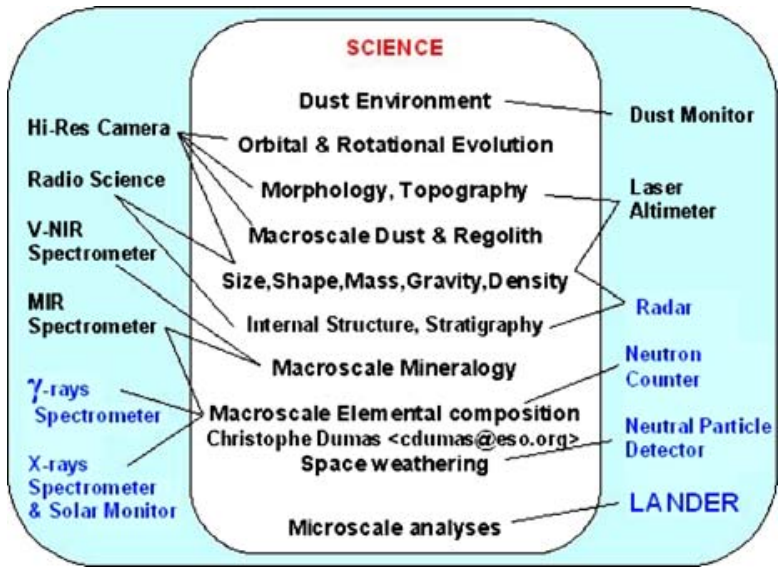

and $100 \times 150 \times 200 \mathrm{~mm}^{3}$. An arm with 3 rotational joints and instruments on the wrist can be proposed.

\section{Basic spacecraft and Lander}

The MSC benefits from the successful development and operation of the Hayabusa spacecraft. The MSC consists of the body and the solar array panel. Attitude is controlled by a three-axis stabilized system. The Lander, re-entry capsule, the ion engines, the sampling devices, the antennas, and the scientific equipments are attached to the body (Fig. 5). The initial total wet mass is $1320 \mathrm{~kg}$ and the payload mass is $200 \mathrm{~kg}$ including the Lander and re-entry capsule. Estimated masses are shown in Table 3 . The size of the MSC body is about $1500 \times 2200 \times 1500 \mathrm{~mm}$.

For the samples collected on the surface by the "touch and go" strategy of the MSC (a direct heritage of successful touchdowns performed by JAXA's Hayabusa spacecraft on the S-type asteroid Itokawa in 2005) two different sampling techniques

Fig. 4 Lander instruments which will allow to meet the scientific objectives. In black the primary payload, in blue the secondary priority

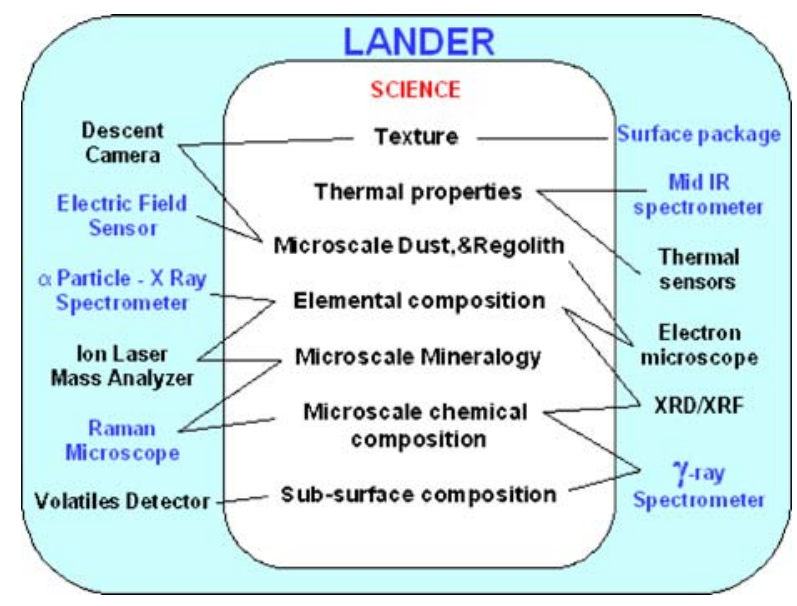




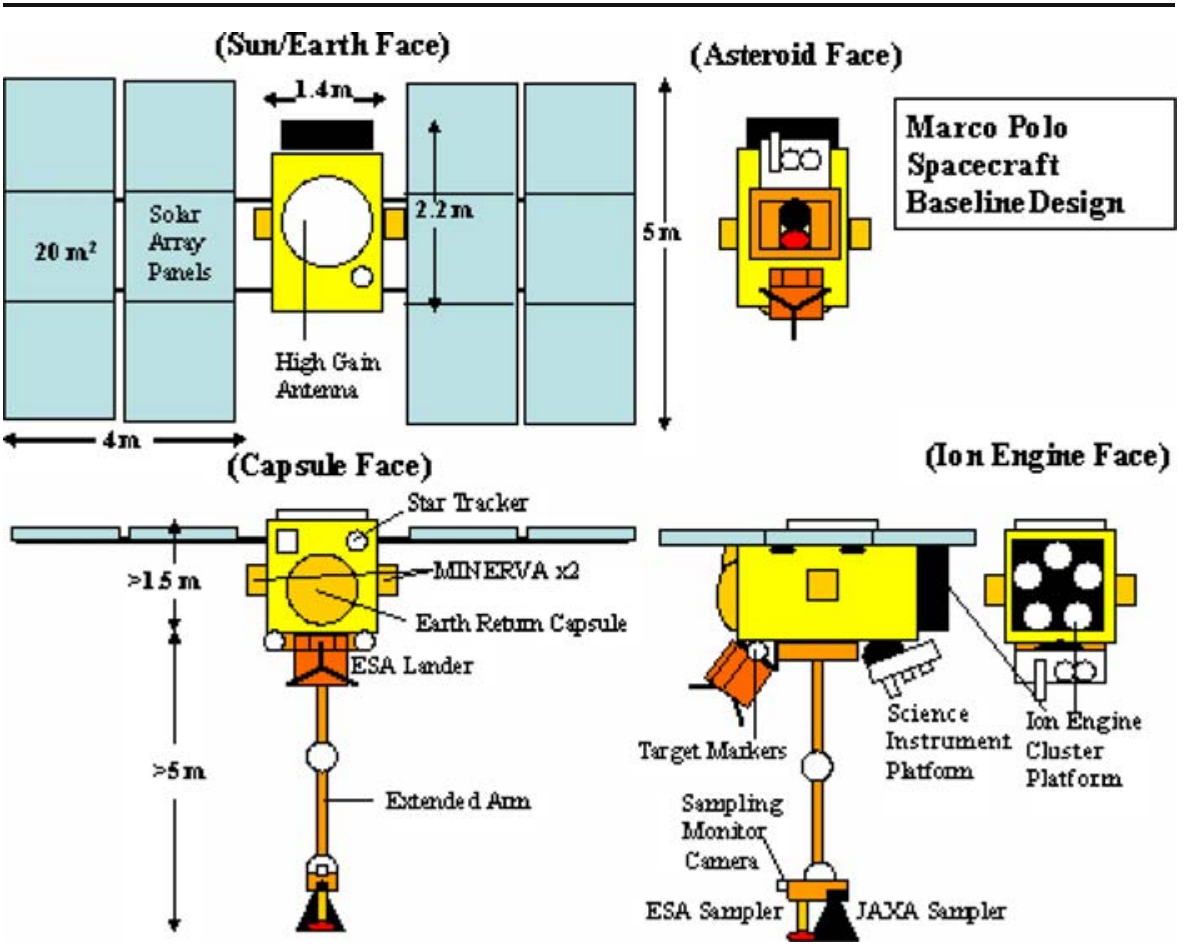

Fig. $5 \mathrm{MSC}$ views, which benefits from the successful experience of the Hayabusa spacecraft development

will be mounted on a retractable extension arm, based on a concept developed by Northrop Grumman. The sampling manoeuvres are performed using a combination of the navigation camera, LIDAR, laser range finders, fan beam sensors, target makers, and touchdown sensors of the sampler horn.

The length of the arm carrying the two sampling devices will be at least equivalent to the satellite's size for safety. One sampling technique developed in Japan will use the heritage from the Hayabusa technique. The other concept from Europe will probably be based on the use of a sticky pad (Fig. 6), which is a very

Table 3 Mass of spacecraft (heritage Hayabusa)

\begin{tabular}{lr}
\hline Components & Mass (kg) \\
\hline Ion Engine System & \\
Xe & 310 \\
System & 230 \\
RCS (Reaction Control Sys.) & 60 \\
BP Fuel & 50 \\
System & 150 \\
Solar Array Panel & 200 \\
Structure + Harness + Thermal & 200 \\
Payload + Capsule & 120 \\
Others (Communic.,AOCS..) & 1320 \\
Total & 950 \\
Dry mass & \\
\hline
\end{tabular}


Fig. 6 Example of Sticky pad (diameter $=3 \mathrm{~cm}$ ) able to collect few grams of regolith

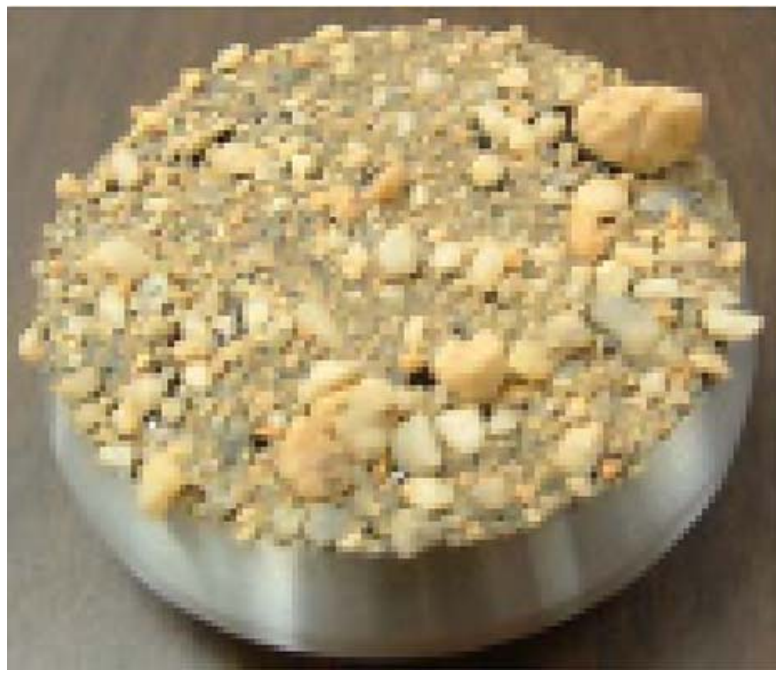

light, robust and simple mechanism. It also has reasonable development time and cost, and due to micro-gravity, it readily permits collection of samples up to several $\mathrm{cm}$ in size. This simple technique has been studied and tested at JHU/APL [28]. Once the samples have been collected, the arm will be used to transfer them to the MSC.

Once the sample containers are inside the MSC, they are sequentially pushed in the sealing system and into the ERC. Then the latch and ERC sealing is performed.

The attitude of the MSC is controlled by Reaction Wheels (RW). There are four RWs, one of them being used as backup. The control procedure is zero-momentum method and the accuracy of the attitude will be $0.1^{\circ}$.

The MSC has small chemical thrusters (RCS: Reaction Control System), which will be used for orbital manoeuvres and unloading of RWs. The main thruster system during the cruise phase is the ion engine system (IES).

In order to determine the attitude, the solar sensor and the star tracker are used. The MSC also has an altimeter, optical navigation cameras, and several sensors for navigation near the NEO.

We plan also to have one or two small rovers (Minerva heritage) of about $10 \mathrm{~cm}$ on the MARCO POLO spacecraft in order to have some characterization of the surface in different places than the Lander site. See Fig. 7 in which the artistic view shows the MSC with the arm for sampling, the Lander and a Minerva hopper.

We adopt the m20 ion engine of JAXA instead of the m10, which was used on the Hayabusa spacecraft. The $\mathrm{m} 20$ is a $20 \mathrm{~cm}$ diameter, $30 \mathrm{mN}, 1 \mathrm{~kW}$ ion engine, which is under development by JAXA. The ISP of this ion engine is about $3000 \mathrm{~s}$. There are 5 engine heads, providing redundancy. Each engine is driven by $1 \mathrm{HV}$ power supply of $1 \mathrm{~kW}$ class.

The Solar Array Panel assumed here is 3.5 times as large as that of Hayabusa, and it generates $9 \mathrm{~kW}$ at a distance of $1 \mathrm{AU}$ from the Sun and $2.5 \mathrm{~kW}$ at 2 AU. The number of ion engines in operation depends on the distance from the Sun. When there is enough power, 4 ion engines are operated simultaneously, but when the 
Fig. 7 Artistic view of the MSC with the deployed arm, the Lander Sifnos, based on the Rosetta Lander Philae heritage and a Minerva hopper

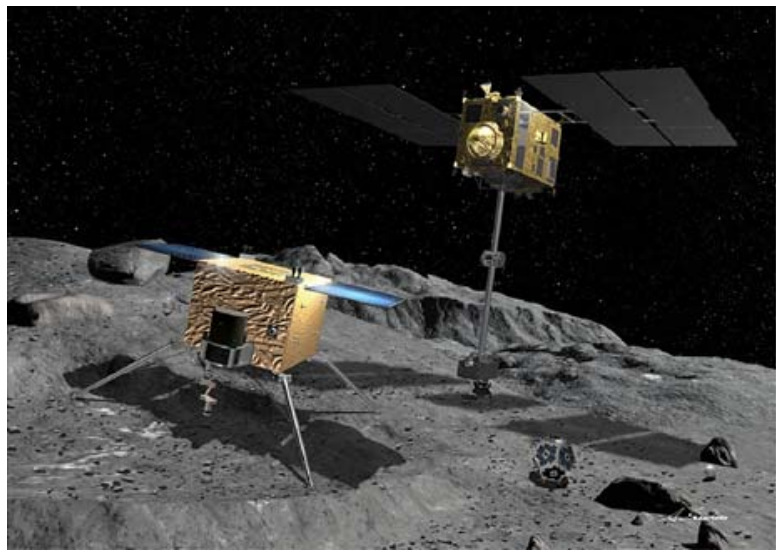

power is low, the number of ion engines will be limited. Throttling operation is also considered when the power is low.

The data transmission is based on the CCSDS (Consultative Committee for Space Data Systems). The commands and the telemetry are processed by the DHU (Data Handling Unit). The subsystems interface with DHU by the PIM (Peripheral Interface Module).

The communications between the MSC and the ground stations are done via Xband and Ka-band. The daily operation of the MSC is mainly performed by X-band, and occasionally Ka-band is used to download the scientific data. The bit rate, which was $8 \mathrm{kbit} / \mathrm{s}$ at most in case of the Hayabusa spacecraft will be larger by more than one order of magnitude for MARCO POLO.

The operations of the MSC are carried out from SSOC (Sagamihara Space Operation Center of JAXA) in Japan. In order to communicate with the spacecraft, the $64 \mathrm{~m}$ antenna of UDSC (Usuda Deep Space Center) in Japan will be used. When 24 hour tracking will be needed (NEO RdV, Lander release, sampling....) the tracking station of ESA will also be used (for emergency: NASA DSN tbc). The telemetry data will be received in SSOC and will also be transferred to ESA.

The Lander, named SIFNOS, will be provided by ESA. It will operate a few days on the NEO surface. The in situ investigation is considered particularly important to identify i) the context where the samples are taken (both the ones collected by the MSC and that collected by SIFNOS), ii) the heterogeneity of the sampling area, and iii) any contamination/modification due to the sample transfer to Earth.

The Lander itself profits considerably from the Rosetta Lander (Philae) heritage. Philae is a $\sim 100 \mathrm{~kg}$ Lander (incl. about $26 \mathrm{~kg}$ of scientific payload), designed to land on a comet nucleus. The requirements regarding separation, descent and landing of the Rosetta Lander on the comet's surface are comparable to those for landing on an NEO. Figure 7 shows an artists impression of the Lander, with its landing gears to dissipate most of the impact energy (to avoid rebounding). Anchoring harpoons will guarantee good fixation to the ground like in the case of Philae.

An estimate of the mass breakdown for the Lander SIFNOS and its sampling device is indicated in Table 4. 
Table 4 Mass breakdown for Lander (excluding units on MSC)

\begin{tabular}{lrcc}
\hline & $\begin{array}{l}\text { Mass } \\
(\mathrm{kg})\end{array}$ & $\begin{array}{l}\text { Margin } \\
\%\end{array}$ & $\begin{array}{l}\text { Mass + margin } \\
(\mathrm{kg})\end{array}$ \\
\hline Structure & 18 & 30 & 23.4 \\
Thermal control Syst. & 7 & 25 & 8.75 \\
Power (incl. Batteries) & 15 & 25 & 18.7 \\
Landing System & 12 & 25 & 15 \\
Anchoring System & 2 & 25 & 2,5 \\
CDMS & 3 & 25 & 3,75 \\
TxRx & 2,5 & 20 & 3 \\
Payload + arm & 7,5 & 20 & 9 \\
Total mass & 67 & & 84.1 \\
\end{tabular}

The power system (Table 5) for SIFNOS needs to be sized for science operations during daylight and survival at night. The distance to the Sun during the operational phase and the rotation rate of the NEO are key factors in the power system sizing.

The power demand during the envisaged mission phases, the array sizing and the battery sizing are detailed in Table 5 .

\subsection{Data archive and sample curation approach}

The goals of the Data Archiving and the Sample Curation Facility are to setup, to process and to preserve data obtained and materials collected during the mission, as well as supplementary information acquired in laboratories or ground-based or in orbit observatories. The prime objectives of these activities are:

- to enable and ensure the (long-term) preservation of both the data and the NEO samples

- to distribute of scientifically useful data and materials to the world wide scientific community

- to provide supplementary data services aimed at maximizing the usage of the MARCO POLO mission data and at easing the scientific data analysis.

The returned samples will be the principle resource used to address the scientific goals outlined in this manuscript. However, the amount of material returned will be very limited, of the order of $100 \mathrm{~g}$ at most, and therefore great care has to be taken in ensuring that the samples are properly curated to ensure that the scientific goals of the mission are achieved, and indeed that the science return is maximised.

The breadth of highly specialised measurement requirements means that a large number of laboratories will participate in the analyses of the samples. Equally, any

Table 5 Power modes of the Lander

\begin{tabular}{lcc}
\hline Mode & Power (W) & Power (W) 20\% margin \\
\hline Cruise (years) & 8 & 9.6 \\
$\begin{array}{l}\text { Descent } \\
\quad(5 \text { minutes })\end{array}$ & 36 & 43.2 \\
Touch down & & \\
$\quad(<1$ min) & 71 & 85.2 \\
In situ science & & \\
Eclipse hibernation & 47 & 56.4 \\
& 64 & 76.8 \\
\hline
\end{tabular}


regolith sample from the surface of a NEO will contain multiple lithologies. Accurately cataloguing each of the main components, and distributing the most appropriate samples to each of the participating laboratories are key functions of this facility. This work requires full tracking of sub-sampling to permit subsequent cross referencing of un-expected results, and at the same time to be responsive to minimising contamination for many different types of analyses.

The facility will require a number of analytical tools, particularly those which are non-destructive or with little or no sample requirement - including microscopy and spectroscopy - to ensure that the most appropriate samples are distributed to each lab. Dedicated sample processing facilities will also be required to ensure that optimised quantities of material can be selected for each analysis program and that specific and general contamination levels are attained.

Current COSPAR planetary protection policy does not clearly indicate whether high levels of containment are required for this mission in order to meet planetary protection constraints.

One of the great benefits of a sample return is that material can be retained for future generations to address scientific problems not known at the present time or to apply new analytical techniques that offer greater insight into processes that we can currently investigate. Therefore, a significant portion of the returned sample should be retained for future study, demanding that some level of operation of the curation facility will be required for many years. Other applications for this facility could of course potentially include future Mars SR and any other materials returned by ESA, JAXA, or national agencies.

Considerable expertise in the curation of extra-terrestrial materials, particularly working with clean environments as well as some aspects of planetary protection, already exists within a number of European centres. Therefore, development of such a facility within Europe can be readily supported from local expertise. In addition, Japanese partners also have extensive expertise in such matters, including preparation for return of Haybusa samples.

The Stardust and Genesis missions brought back to Earth microgram quantities of sample, which required the participating laboratories to further develop microanalysis techniques such as X-ray synchrotron mineralogy, electron microscopy, ion probe, laser ablation-static mass spectrometry, etc. European laboratories continue to play a major role in the study of these important samples, and in anticipation of the successful return of Marco Polo it is important that Europe retains such world class facilities with appropriate expertise in the micro- and nano- characterization and analysis of ET material. These facilities are routinely applied to the analysis of meteoritic materials and span the entire breadth of expertise that is required for this mission - including feeding into the sample requirements that the sample curation facility must consider in storage, handling and distribution.

\section{Key technology areas}

Cathode-less Microwave Discharge Ion Engines The $\mathrm{m} 10$ ion engines generate plasmas without any cathodes, which are life critical parts, so that they are durable and reliable for space operations. They were validated by the powered flight of 
Hayabusa between Earth and the asteroid in deep space. The $\mathrm{m} 20$ ion engines succeeding to the m10's space heritage have a capability of $30 \mathrm{mN}$ thrust with $3000 \mathrm{sec}$ specific impulse reinforced from $8 \mathrm{mN}$.

Re-entry Capsule In MARCO POLO, the re-entry velocity of the return capsule from the baseline target Wilson-Harrington will be 2 to $2.5 \mathrm{~km} / \mathrm{s}$ higher than the $12 \mathrm{~km} / \mathrm{s}$ of the Hayabusa mission. Though the consequent flight environment experienced by the capsule will be severe, it can be tolerated adapting the present thermal protection technology of the Hayabusa mission by selecting optimised reentry flight path angles or by designing an appropriate thickness of the insulator of the heat shield. Nevertheless, by adopting novel lightweight ablators, it may even be possible to reduce the heatshield mass. The super lightweight ablators are now under development, and the computational analysis technology has also made some progress in these last years after the design completion of Hayabusa. The development of the lightweight heat shield, in particular the actual innovative thermal protection material, will be significant for the future space exploration programs of both Japan and Europe. A common ESA-JAXA study on high speed reentry capsules has already started.

Communication Low phase noise small Ka-band transponders (or transmitters) will be available by the end of the next decade. Highly efficient Ka-band power amplifiers and light-weight high-gain planar antennas will be developed in parallel. Deep space activities aim at being enhanced by using the Ka-band technology in next generation ground stations. As part of station capabilities, new coding schemes such as turbo and LDPC (Low Density Parity Check) coding will be implemented to support higher data rate. Additionally, DDOR (Differential Doppler Ranging) for Ka-band will be introduced. The basic study to realize Ka-band technologies has been partially done and simulated. The development of inter-spacecraft communication equipment to guarantee a stable link among them will also be established based on the undergoing formation flight technology.

Sampling from MSC The descent of the two sampling devices from the MSC to the surface will be performed by an extensible arm, such as the bi-STEM of Northrop Grumman. This arm will also be used to retrieve the samples to the MSC. While a STEM has already been used in extension phases in previous space missions (Voyager, HST, Mars Pathfinder ...), it has not been tested yet for a retracting phase. Then, the implementation of a joint absorbing the shock during the touch down at a few $\mathrm{cm} / \mathrm{s}$ is under study.

\section{Conclusions}

MARCO POLO is a joint European-Japanese mission taking advantage of JAXA's pioneering experience acquired with Hayabusa on a small body sample return mission. Concerning the ESA costs, MARCO POLO mission is within the available technical and financial resources of an $\mathrm{M}$ class mission. The internal study phase 
will allow ESA and JAXA to select the best mission scenario between the various proposed options and possible targets.

MARCO POLO mission is innovative and besides its scientific relevance allows: a) testing new technologies; b) preparing and using the next generation of laboratory facilities for extraterrestrial sample analysis; c) pathfinding for future sample returns from high surface gravity bodies.

MARCO POLO is a flexible mission with several possible options and several possible high interest targets. The various phases of the mission are scientifically autonomous and the completion of each of them separately will lead to a major improvement of our knowledge. The returned sample (first for ESA) will be of an inestimable scientific value - particularly for the large sample-analysis community within Europe. In order to be certain that the scientific objectives are met, a specialist sample curation facility will be required to ensure the samples are characterised and distributed appropriately and without adverse terrestrial contamination. The minimum achievement (remote sensing data) will allow us for the first time to reach an understanding of a primitive body comparable to that obtained for the more evolved bodies by previous missions. In situ data, provided by a Lander, will also represent a "premier" in asteroid science.

\section{References}

1. Barucci, M.A., Doressoundiram, A., Fulchignoni, M., Florczak, M., et al.: Search for aqueously altered materials on asteroids. Icarus 132, 388-396 (1998)

2. Barucci, M.A., Capria, M.T., Coradini, A., Fulchignoni, M.: Classification of asteroids using G-mode analysis. Icarus 72, 304-324 (1987)

3. Bernatowicz, T.J., Messenger, S., Pravdivtseva, O., Swann, P., Walker, R.M.: Pristine presolar silicon carbide. Geochim Cosmochim Acta 67, 4679-4691 (2003)

4. Binzel, R.P., Rivkin, A.S., Stuart, J.S., Harris, A.W., Bus, S.J., Burbine, T.H.: Observed spectra properties of near-Earth objects: results for population distribution, source regions, and space weathering processes. Icarus 170, 259-294 (2004)

5. Boss, A.P.: Evolution of the Solar Nebula. VI. Mixing and transport of isotopic heterogeneity. Astrophys. J. 616, 1265 (2004)

6. Boss, A.P., Goswami, J.N.: Presolar cloud collapse and the formation and early evolution of the solar nebula. In: Lauretta, D.S., McSween, H.Y. (eds.) Meteorites and the Early Solar System II, pp. 171186. University of Arizona Press, Tucson (2006)

7. Brown, P.G., Hildebrand, A.R., Zolensky, M.E., Grady, M., et al.: The fall, recovery, orbit, and composition of the Tagish Lake Meteorite: a new type of carbonaceous chondrite. Sciente 290, 320325 (2000)

8. Bus, S.J., Binzel, R.P.: Phase II of the small main-belt asteroid spectroscopic survey. The observations. Icarus. 158, 106-145 (2002a)

9. Bus, S.J., Binzel, R.P.: Phase II of the small main-belt asteroid spectroscopic survey. A feature-based taxonomy. Icarus. 108, 146-177 (2002b)

10. Chapman, C.R.: Space weathering of asteroid surfaces. Annu. Rev. Earth Planet. Sci. 32, 539-567 (2004)

11. Chyba, C.F., Owen, T.C., Ip, W.-H.: Impact delivery of volatiles and organic molecules to earth. In: Gehrels, T. (ed.) Hazards Due to Comets and Asteroids, pp. 9-58. University of Arizona Press, Tucson (1994)

12. Ciesla, F.J.: Outward transport of high-temperature materials around the midplane of the solar nebula. Science. 318, 613-615 (2007)

13. Ciesla, F.J., Charnley, S.B.: The physics and chemistry of nebular evolution. In: Lauretta, D.S., McSween, H.Y. (eds.) Meteorites and the Early Solar System II, pp. 209-230. University of Arizona Press, Tucson (2006) 
14. Donn, B.: The accumulation and structure of comets. In: Newburn, R.L., Neugebauer, M., Rahe, J. (eds.) Comets in the Post Halley Era, pp. 335-359. Kluwer, Dordrecht, the Netherlands (1991)

15. Drake, M.J., Righter, K.: Determining the composition of the earth. Nature 416, 39-44 (2002)

16. Gilmour, I.: Structural and isotopic analysis of organic matter in carbonaceous chondrites. In: Davis, A.M. (ed.) Treatise on Geochemistry, vol. 1, Meteorites, Comets and Planets, pp. 269-290. ElsevierPergamon, Oxford (2003)

17. Hiroi, T., Zolensky, M.E., Pieters, C.M.: The Tagish Lake meteorite: A possible sample from a D-type asteroid.. Science. 293, 2234-2236 (2001)

18. Huss, G.R.: Ubiquitous interstellar diamond and $\mathrm{SiC}$ in primitive chondrites - Abundances reflect metamorphism. Nature 347, 159-162 (1990)

19. Huss, G.R., Lewis, R.S.: Noble gases in presolar diamonds II: Component abundances reflect thermal processing. Meteoritics. 29, 811-829 (1994)

20. Huss, G.R., Lewis, R.S.: Presolar diamond, $\mathrm{SiC}$, and graphite in primitive chondrites: Abundances as a function of meteorite class and petrologic type. Geochim. Cosmochim. Acta. 59, 115-160 (1995)

21. Huss, G.R., Rubin, A.E., Grossman, J.N.: Thermal metamorphism in chondrites. In: Lauretta, D.S., McSween, H.Y. (eds.) Meteorites and the Early Solar System II, pp. 567-586. University of Arizona Press, Tucson (2006)

22. Jacobsen, S.B.: The Hf-W isotopic system and the origin of the Earth and Moon. Annu. Rev. Earth Planet. Sci. 33, 531-570 (2005)

23. Keil, K.: Geological history of asteroid 4 Vesta: The "smallest terrestrial placet". In: Bottke, W.F., Paolicchi, P., Binzel, R.P., Cellino, A. (eds.) Asteroids III, pp. 573-584. University Arizona Press, Tuscon (2002)

24. Keller, L.P., Messenger, S., Flynn, G.J., Clemmett, S., Wirick, S., Jacobsen, C.: The nature of molecular cloud material in interplanetary dust. Geochim. Cosmochim. Acta 68, 2577-2589 (2004)

25. Kleine, T., Münker, C., Metzger, K., Palme, H.: Rapid accretion and early core formation on asteroids and the terrestrial planets from Hf-W chronometry. Nature 418, 952-955 (2002)

26. La Tourette, T., Wasserburg, G.J.: MG diffusion in anorthite: Implications for the formation of early solar system planetesimals. Earth Planet. Sci. Lett. 158, 91 (1998)

27. Lazzarin, M., Marchi, S., Barucci, M.A., Di Martino, M., Barbieri, C.: Visible and near-infrared spectroscopic investigation of near-Earth objects at ESO: First results. Icarus 169, 373-389 (2004)

28. Lees, J., Chabot, N., Persons, D., Hartka, T.: Small body sampling techniques being developed at Johns Hopkins University Applied Physics Laboratory. IAC 06-A3.5.04 (2006)

29. Levison, H.F., Morbidelli, A., Gomes, R., Backman, D.: Planet migration in planetesimal disks. In: Reipurth, B., Jewitt, D., Keil, K. (eds.) Protostars and Planets V, pp. 669-684. University of Arizona Press, Tucson (2006)

30. Lunine, J.I.: Origin of water ice in the solar system. In: Lauretta, D.S., McSween, H.Y. (eds.) Meteorites and the Early Solar System II, pp. 309-219. University of Arizona Press, Tucson (2006)

31. Maurette M. Micrometeorites and the mysteries of our origins. Springer-Verlag, Berlin/Heidelberg (2005)

32. Michel, P., Migliorini, F., Morbidelli, A., Zappalà, V.: The population of Mars-crossers: Classification and dynamical evolution. Icarus 145, 332-347 (2000)

33. Morbidelli, A., Jedicke, R., Bottke, W.F., Michel, P., Tedesco, E.F.: From magnitudes to diameters: the albedo distribution of near Earth objects and the Earth collision hazard. Icarus 158, 329-342 (2002)

34. Morbidelli, A., Gounelle, M., Levison, H.F., Bottke, W.F.: Formation of the binary near-Earth object 1996 FG3: Can binary NEOs be the source of short-CRE meteorites? Meteorit. Planet. Sci. 41, 875887 (2006)

35. Meyer, B.S., Zinner, E.: Nucleosynthesis. In: Lauretta, D.S., McSween, H.Y. (eds.) Meteorites and the Early Solar System, pp. 69-108. Univ Arizona Press, Tucson (2006)

36. Nguyen, A.N., Zinner, E.: Discovery of ancient silicato stardust in a meteorite. Sciente. 303, 14961499 (2004)

37. Nichols, R.H.: Chronological constraints on planetesimal accretion. In: Lauretta, D.S., McSween, H.Y. (eds.) Meteorites and the Early Solar System II, pp. 463-472. University of Arizona Press, Tucson (2006)

38. Noble, S.K., Pieters, C.M., Keller, L.P.: An experimental approach to understanding the optical effects of space weathering. Icarus 192, 629-642 (2007)

39. Perozzi, E., Rossi, A., Valsecchi, G.B.: Basic targeting strategies for rendezvous and flyby missions to the near-Earth asteroids. Planet. Space Sci. 49, 3-22 (2001)

40. Pieters, C.M., Taylor, L.A., Noble, S.K., Keller, L.P., Hapke, B.H., Morris, R.V., Allen, C.C., McKay, D.S., Wentworth, S.: Space weathering on airless bodies: Resolving a mystery with lunar samples. Meteorit. Planet. Sci. 35, 1101-1107 (2000) 
41. Pizzarello, S., Cronin, J.R.: Non-racemic amino acids in the Murray and Murchison meteorites. Geochim. Cosmochim. Acta 64, 329-338 (2000)

42. Pizzarello, S., Cooper, G.W., Flynn, G.J.: The nature and distribution of of organic material in carbonaceous chondrites. In: Lauretta, D.S., McSween, H.Y. (eds.) Meteorites and the Early Solar System, pp. 625-651. Univ Arizona Press, Tucson (2006)

43. Remusat, L., Palhol, F., Robert, F., Derenne, S., France-Lanord, C.: Earth Planet. Sci. Lett. 243, 15-25 (2006)

44. Righter, K., Drake, M.J., Scott, E.: Compositional relationships between meteorites and terrestrial planets. In: Lauretta, D.S., McSween, H.Y. (eds.) Meteorites and the Early Solar System II, pp. 803828. University of Arizona Press, Tucson (2006)

45. Stuart, J.S., Binzel, R.P.: Bias-corrected population, size distribution, and impact hazard for the nearEarth objects. Icarus 170, 295-311 (2004)

46. Weidenschilling, S.R.: The Origin of Comets in the Solar Nebula: A Unified Model. Icarus 127, 290306 (1997)

47. Weissman, P.R., Bottke, W.F., Levison, H.F.: Evolution of comets into asteroids.. In: Bottke, W.F., Cellino, A., Paolicchi, P., Binzel, R.P. (eds.) Asteroids III, pp. 669-686. The University of Arizona Press, Tucson (2002)

48. Zolensky, M.E., 75 co-authors: Mineralogy and petrology of comet 81P/Wild 2 nucleus samples. Science 314, 1735-1739 (2006) 